\title{
Adherence Support Approaches in Biomedical HIV Prevention Trials: Experiences, Insights and Future Directions from Four Multisite Prevention Trials
}

\author{
K. Rivet Amico - Leila E. Mansoor • Amy Corneli • \\ Kristine Torjesen $\cdot$ Ariane van der Straten
}

Published online: 23 February 2013

(C) Springer Science+Business Media New York 2013

\begin{abstract}
Adherence is a critical component of the success of antiretroviral-based pre-exposure prophylaxis (PrEP) in averting new HIV-infections. Ensuring drug availability at the time of potential HIV exposure relies on self-directed product use. A deeper understanding of how to best support sustained PrEP adherence remains critical to current and future PrEP efforts. This paper provides a succinct synthesis of the adherence support experiences from four pivotal PrEP trials-Centre for the AIDS Programme of Research in South Africa (CAPRISA) 004, FEM-PrEP, Iniciativa Prophylaxis (iPrEx), and Vaginal and Oral Interventions to Control the Epidemic (VOICE). Notwithstanding variability in the design, population/cohort, formulation, drug, dosing strategy, and operationalization of adherence approaches utilized in each trial, the theoretical basis and experiences in implementation and monitoring of the approaches used by these trials provide key lessons for optimizing adherence in
\end{abstract}

\section{K. R. Amico ( $\square)$}

Center for Health Interventions and Prevention,

University of Connecticut, Storrs, CT, USA

e-mail: rivetamico@comcast.net

\section{E. Mansoor}

Centre for the AIDS Programme of Research in South Africa (CAPRISA), University of KwaZulu-Natal, Durban,

South Africa

\section{A. Corneli · K. Torjesen}

FHI 360, Durham, NC, USA

\section{A. van der Straten}

Women's Global Health Imperative, RTI International

(Research Triangle Institute), San Francisco, CA, USA

A. van der Straten

Department of Medicine, Center for AIDS Prevention Studies,

University of California San Francisco, San Francisco, CA, USA future research and programmatic scale-up of PrEP. Recommendations from across these trials include participantcentered approaches, separating measurement of adherence from adherence counseling, incorporating tailored strategies that go beyond education, fostering motivation, and addressing the specific context in which an individual incorporates and negotiates PrEP use.

\begin{abstract}
La adherencia al producto es un componente crítico para el éxito de la pre-exposición profiláctica (PrEP, siglas en inglés) basada en antirretrovirales para prevenir nuevas infecciones del VIH. Asegurar la presencia del medicamento cuando existe la posibilidad de exposición al VIH depende en el uso autodirigido del producto. Un mejor entendimiento de cómo mejor apoyar adherencia sostenida a PrEP sigue siendo crítico en los esfuerzos presentes y futuros de PrEP. Este papel provee un sucinto resumen de las experiencias de apoyo a la adherencia de cuatro estudios esenciales -CAPRISA 004 (Centre for the AIDS Programme of Research in South Africa, FEMPrEP, Iniciativa Prophylaxis (iPrEX), y VOICE (Vaginal and Oral Interventions to Control the Epidemic). A pesar de la variaciones en los diseños, poblaciones/cohortes, fórmulas, drogas/medicamentos, estrategias de dosis y operacionalización de enfoques de adherencia utilizadas en cada estudio, las bases teóricas y experiencias en implementación y monitoreo de los enfoques utilizados por estos estudios proveen lecciones claves para optimizar la adherencia en futuros estudios y la ampliación programática de PrEP. Las recomendaciones de todos estos estudios incluyen enfoques centralizados en los participantes, separando medidas de adherencia de la consejería sobre la adherencia, incorporando estrategias adaptadas que van mas allá de la educación, fomentando la motivación y dirigiendo el contexto específico en el cual un individuo incorpora y se adapta al uso de PrEP.
\end{abstract}


Keywords PrEP - Microbicides · Adherence .

Intervention · Counseling · Clinical trials · HIV prevention

\section{Introduction}

Efforts to reduce HIV infection through the use of topical and oral prophylactic antiretrovirals (ARVs) have recently yielded remarkable successes [1-4], with two exceptions. In the FEM-PrEP trial, product adherence was too low to assess the efficacy of daily tenofovir disoproxil fumarate/ emtricitabine (TDF/FTC, Truvada) in preventing HIV acquisition in the study population [5]. In the Vaginal and Oral Interventions to Control the Epidemic (VOICE) trial, futility results (the inability to show efficacy for protection from HIV infection) for daily oral tenofovir and vaginal tenofovir gel remain to be explained [6,7]. The FDA approval of prophylactic use of Truvada has increased attention on who should be the target group for oral PrEP and how to optimize PrEP adherence to maximize its prevention potential. Even with highly effective ARVbased PrEP drugs, use of the drug is essential to confer protection, and no or low drug use results in no protection $[5,8]$. In both the Iniciativa Prophylaxis (iPrEx) and Centre for the AIDS Programme of Research in South Africa (CAPRISA) 004 trials, a clear dose response could be delineated with higher levels of protection observed with higher adherence to product use. Enhancing understanding of who will use PrEP and how it will be used is recognized as an important challenge $[9,10]$. In contrast to the large body of literature available with respect to supporting and promoting adherence to ARVs in a therapeutic context, less is known about how to support adherence in the context of prophylactic use of ARVs among healthy, HIV-uninfected individuals [11, 12]. Despite clear recommendations to support or 'counsel on' PrEP adherence among those opting to use it [13-15], to date there are few publications or guidelines on how to operationally and effectively do so.

Evidence-based guidance on how to best support PrEP adherence is starting to emerge [12], as this area of research has only recently come to life with the identification of effective PrEP products. All blinded trials of PrEP agents have used strategies to support adherence in their study populations, and all are confronted with the challenges of promoting adherence among healthy participants to products of unknown efficacy, incomplete safety profiles, and with equal odds of participants receiving the active agent or a placebo. Despite the limitations of product use in clinical trial settings, lessons learned from the approaches used to support product use in blinded trials can offer concrete guidance for future PrEP efficacy trials, and initial guidance for supporting PrEP use in "real life" contexts.
Here, we offer an overview of the adherence support approaches developed for use in CAPRISA 004, FEMPrEP, iPrEx, and VOICE (detailed in Table 1). Our aim is to describe the approaches deployed, clarify the strategies leveraged to promote product use in these diverse settings, and share experiences that will likely continue to be relevant in demonstration projects. Within each trial, the adherence support approach was applied as standard practice. Hence, evaluation of adherence intervention efficacy with adequate comparison controls is not possible. Rather, our explorations are based on experiences amassed by study teams and the resulting recommendations that emerge from these for supporting PrEP use in demonstration and efficacy trials.

\section{CAPRISA 004}

\section{Overview}

CAPRISA 004 was a phase IIb randomized controlled trial (RCT) assessing the safety and effectiveness of $1 \%$ tenofovir gel to prevent HIV infection in sexually active HIVuninfected South African women [1]. A coitally linked dosing strategy was used wherein participants were counseled to use the study gel before and after sex (also referred to as BAT24-Before and After sex, within $12 \mathrm{~h}$ and not to exceed Two doses in $24 \mathrm{~h}$ ). Results from this trial demonstrated $39 \%$ effectiveness of tenofovir gel to reduce risk of HIV acquisition, with $54 \%$ protection among high adherers (defined as the women with $>80 \%$ of coital acts with before-and-after-gel use, based on gel applicator return and reported sexual events) [1].

Adherence Support Approach: Customized Adherence Support Program (ASP)

Adherence activities evolved over three distinct stages: pre-trial, months $0-15$, and months 16-30. The ASP program was implemented in the latter trial stage; however, it drew heavily from the education and skillsbuilding already implemented with all participants in the pre-trial and early-trial stages. During the pre-trial phase a demographic and health survey was undertaken to establish community understanding of research, key concepts relating to informed consent and randomized controlled trials, and rights of participants. These data were used to develop the informed consent process, establish HIV incidence rates in the proposed study sites, and assess HIV risk factors and their desired dosing strategies. Given that the majority of the volunteers had partners who were migrant workers and coital 


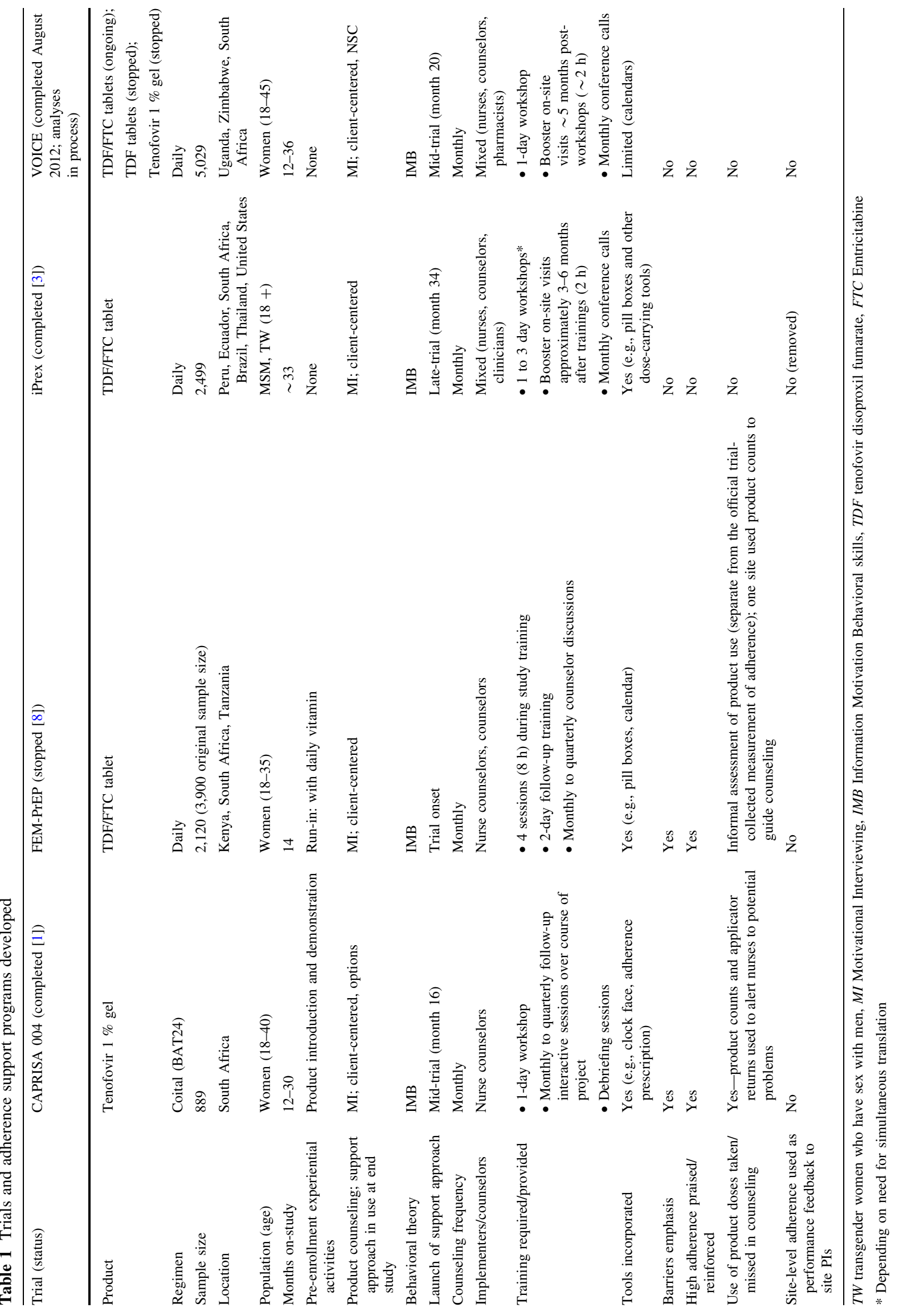


activity was linked to those days when the partner was home, volunteers were keen on a product that could be used around coitus, which led to the development of the BAT24 strategy.

The adherence support approach implemented from initiation through mid-trial (months 0-15) emphasized the mechanics of gel use, understanding of dosing regimen, and development of strategies for integration of gel use into daily activity. These efforts were guided by the Information-Motivation-Behavioral skills (IMB) model [16] and delivered by staff who had received extensive training based on potential user profiles identified in the pre-trial initiation stage and on an ongoing basis based on implementation. Narrative scripts were expanded as the cohort increased and new scenarios for product use emerged. General information sessions were held for groups of potential participants, and included an opportunity to view the gel applicator. Potential participants were provided with samples of demonstration gel to get a sense of its texture, odor, and consistency. At enrollment, participants were provided with detailed information on the mechanics of gel use, including how to assemble the applicator and how to insert the applicator and eject the gel into the vagina. The dosing strategy was discussed in detail and strategies to incorporate gel use into daily activities were explored. These information sessions were supplemented with demonstrations using flipcharts, a clock-face pictogram, and opportunities to manipulate the applicator and practice correct insertion using a female pelvic model. At each monthly scheduled study visit, gel use was assessed using a structured interviewer-administered questionnaire. Detailed data were collected on study product use for their most recent coital day, 7 days prior to their last coital day, and in the past month. The nurse who collected this data used the information to provide additional counseling to support any aspect of gel use that might be posing a challenge for participants in the previous month. This assessment was supplemented with pharmacy counts of the returned used and unused applicators.

Towards the latter part of year 1, a pharmacist counting the returned applicators noted that some applicators were being returned from another microbicide trial, and alerted the study team. The root cause analysis [17] determined that a number of participants had co-enrolled in another trial; this also raised concerns about inaccuracies in or misrepresentation of product use more generally. In response, the study team conducted a careful evaluation of the adherence assessment and support approach currently implemented, with emphasis on strategies in place that may challenge accurate reporting of gel-use and alternative strategies that could better support reporting and actual use. A series of discussions were conducted with study staff and trial participants that asked specific questions about their perceptions of the negative consequences of reporting nonadherence, and whether procedures that were already in place would deter the open discussion of challenges to product use. These, in addition to consultations with a number of behavioral scientist experts in adherence and health behavior, produced the following key recommendations: (a) separation of adherence assessment from adherence support counseling (use of different staff for adherence support versus adherence measurement); (b) shift in focus from the mechanics of gel use and adherence to the BAT2 4 dosing strategy to identification of barriers and facilitators of gel use; and (c) non-judgmental, customized adherence support to address challenges faced with product use in the past month. Based on these recommendations the study team revised the adherence activities and incorporated several elements of the Options Project, an intervention based on motivational interviewing (detailed in [18]). The resulting customized adherence support program (referred to as ASP) was then implemented for the remainder of the trial.

From month 16 to month 30, ASP was implemented with the use of a Motivational Interviewing (MI) approach to facilitate a brief, participant-centered, collaborative discussion; resolve ambivalence around adherence; and help the participant develop a product-use plan that would work for her $[19,20]$. This shifted from a predominantly assessmentdriven and education-based approach to one that targeted the participant's motivation to use the product. Measurement of product use was removed from the counseling discussion, and was collected by a different staff member.

The main steps of the ASP are presented in Fig. 1. A key component of the ASP is the participant-centeredness. Instead of reinforcing the product dosing strategy, the focus was on use of product in relation to planned or anticipated coital activity and participants developing their own plans for incorporating the product into their routine. Incremental goal setting was introduced and supported over a period of time towards enhancing BAT24 adherence.

\section{Monitoring and Evaluation}

Regular debriefing sessions were held with the nurse counselors. This team-based implementation support approach facilitated the sharing of successful strategies, and appeared to promote the adoption of ASP by staff. At the end of the CAPRISA 004 trial, adherence levels achieved using the ASP approach were compared to those attained pre-ASP. The most reliable marker to measure product use was returned used applicators [1]. More than $95 \%$ of applicators dispensed were returned either as used or unused, which suggests the feasibility of using product return in clinical trials to measure adherence. Based on returned used applicators and reported coital acts, 


\begin{tabular}{|c|c|}
\hline 1 & $\begin{array}{l}\text { SET THE AGENDA } \\
\text { Tell participant about content of discussion and ask permission to continue discussion }\end{array}$ \\
\hline 2 & $\begin{array}{l}\text { ASSESS GEL USE } \\
\text { Evaluate gel use since last visit and at last sex event }\end{array}$ \\
\hline 3 & $\begin{array}{l}\text { ASSESS IMPORTANCE OF CORRECT GEL USE AND CONFIDENCE IN ABILITY TO USE GEL } \\
\text { On a scale from } 1 \text { to } 10 \text {, rate importance of and/or confidence in using gel }\end{array}$ \\
\hline 4 & $\begin{array}{l}\text { IDENTIFY BARRIERS } \\
\text { Focus on improving importance or confidence by identifying barriers to either }\end{array}$ \\
\hline 5 & $\begin{array}{l}\text { DISCUSS STRATEGIES } \\
\text { Identify potential strategies for barriers resolution }\end{array}$ \\
\hline 6 & $\begin{array}{l}\text { SUMMARIZE AND SET GOAL } \\
\text { Ask participant to identify a strategy to use }\end{array}$ \\
\hline 7 & $\begin{array}{l}\text { NEGOTIATE ACTION PLAN } \\
\text { Discuss how strategy will be implemented }\end{array}$ \\
\hline 8 & $\begin{array}{l}\text { DOCUMENT } \\
\text { Document selected strategy as a prescription plan for participant. }\end{array}$ \\
\hline
\end{tabular}

Fig. 1 Steps and framing of the ASP

participants were categorized into one of three levels of adherence: high BAT24 use of gel $(>80 \%)$, intermediate BAT24 use of gel (50-80\%) and low BAT24 use of gel $(<50 \%)$. The ASP increased the number of intermediate users to high, but did not have an impact on low adherers [21]. Drug levels in genital specimens correlated with returned used applicators, so that the highest drug levels were found in the high adherence group and the lowest levels were found in the low adherence group [8].

\section{FEM-PrEP}

\section{Overview}

FEM-PrEP was a phase III RCT designed to assess the safety and effectiveness of a daily oral dose of TDF/FTC for HIV prevention among women at higher risk of HIV in Kenya, South Africa, and Tanzania (see Table 1). In April 2011, the FEM-PrEP investigators decided to stop the trial early, because the Independent Data Monitoring Committee recommended that the study would be unlikely to demonstrate the effectiveness of TDF/FTC in preventing HIV infection even if the trial continued to its planned end and all end-points were collected [5].
Prior to trial initiation, socio-behavioral research was conducted to inform the behavioral components of the trial [22]. In-depth interviews (IDIs) and focus group discussions (FGDs) were conducted with potential participants, community stakeholders, and men from the community to identify factors that might facilitate or impede daily pill use. These data guided the development of the adherence counseling approach for FEM-PrEP participants, as did adult learning principles, lessons learned from adherence counseling for antiretroviral treatment and contraceptives, the IMB model, and MI [23-30]. In addition, a vitamin runin period was implemented prior to dispensation of study drug. The vitamin was about the same shape and size as the study tablets. Participants were asked to take one vitamin daily between the screening and enrollment visits. At enrollment, participants were observed swallowing the tablet. Those unable to swallow the tablet could not enroll. Experiences with vitamin adherence were then explored during adherence counseling at enrollment and used to create personal adherence plans for the study drug.

\section{Adherence Support Approach}

Participant-centered and goal-oriented adherence counseling was provided by a trained counselor at each monthly 
study visit in all sites as early as possible in the flow of the visit. Participants developed a personal adherence plan in collaboration with the study counselors, which included strategies to integrate pill-taking into daily routines and social context. Each follow-up adherence counseling session began with participants describing their experience with trying out their previous adherence goal over the past month. Counselors also asked participants how many pills they missed in the past 7 days. Participants described any barriers they faced and how useful the previously identified strategies had been in overcoming those barriers. Counselors tailored the counseling topics and questions to each participant's situation, although the same steps were followed for each participant regardless of their reported level of adherence. Counselors were trained to remain nonjudgmental when instances of non-adherence were reported, and to acknowledge good or improved adherence. Counselors were also trained to assist participants in transforming barriers into goals. For example, if a participant had difficulty taking the pills over the weekend, the participant and counselor would strategize on how the participant might be able to take the pills during those days (e.g., set an alarm, link to an event that occurs on weekends), and the goal would focus on the implementation of that strategy. Counseling ended with participants refining their adherence plans and goals as needed. Table 2 lists specific steps followed at each counseling session.

Adherence counseling checklists were used to facilitate counseling in all sites, and prior to the counseling session the counselors reviewed the checklist from the previous visit in order to contextualize the current counseling session. Various adherence tools were also offered (e.g., pill boxes, calendars), and key messages on adherence were provided at strategic time points over the course of the trial to provide necessary information but to avoid overburdening participants with too much information (e.g., key messages were explained in detail at enrollment, assessed and summarized monthly for the first quarter, and then provided on an as-needed basis thereafter).

Operational guidance was also provided. In some sites, IDIs were conducted quarterly with a $5 \%$ random sample of enrolled participants. Data describing the adherence facilitators, barriers, and experiences with the study product among these participants, as well as summaries of selfreported adherence among all participants, were provided to counselors so that they could explore the content with participants when applicable (e.g., suggestions on facilitators, experience with specific barriers, pill taking fatigue). In addition, in 2010 one site expressed concerns that adherence may be lower than reported, following feedback from various community forums and information obtained from participants during the IDIs. In response, all participants at that site were invited to take part in one of several group meetings to discuss concerns about adherence and rumors about the study product. Participants' concerns were summarized and provided to counselors; counselors were encouraged to explore with participants in subsequent counseling sessions the possible rumors that may have impeded their pill use.

To reduce socially-desirable responses in the self-report measurement of adherence, the behavioral questionnaires were administered before adherence counseling by different staff from those facilitating adherence counseling in all sites. Counselors did not review these behavioral questionnaires prior to providing counseling. ${ }^{1}$

\section{Monitoring and Evaluation}

Observations of adherence counseling were conducted by behavioral leadership at some sites. Discussions among counselors took place monthly after trial initiation, and then quarterly to review data summaries, discuss experiences, and make decisions on steps to enhance adherence counseling as appropriate. Data were collected during IDIs with women who seroconverted during the trial on their perceptions of adherence counseling. These data are currently being analyzed.

Primary analyses revealed that adherence was low, despite the substantial adherence counseling support described above. Fewer than $40 \%$ of a representative subsample of uninfected women had any evidence of study drug in plasma within $48 \mathrm{~h}$ of specimen collection. In contrast, self-reported adherence was high, with $95 \%$ of participants reporting that they 'usually' or 'always' used the product as recommended over the course of the trial, and pill count data were consistent with participants having used the product on $88 \%$ of days in the trial [5].

Additional drug level analyses of stored biological specimens are planned in order to characterize adherence among study participants assigned to TDF/FTC. Specifically, intracellular drug level testing will provide a better understanding of adherence in between study visits since tenofovir diphosphate has a half-life measured in days and remains detectable weeks after cessation of dosing. Mixedmethod analyses integrating quantitative, qualitative, and drug level data will also provide insight into low adherence, discrepancy between self-report and drug level data, and variations across sites. These analyses will examine the association between HIV risk perception and drug level, facilitators and barriers to adherence among those with various drug levels, population characteristics and

\footnotetext{
1 At the request of one site, counselors counted pills in front of participants at the beginning of adherence counseling in order to have a better understanding of adherence to guide counseling.
} 
Table 2 FEM-PrEP: Adherence counseling topics at the screening, enrollment, and follow-up visits

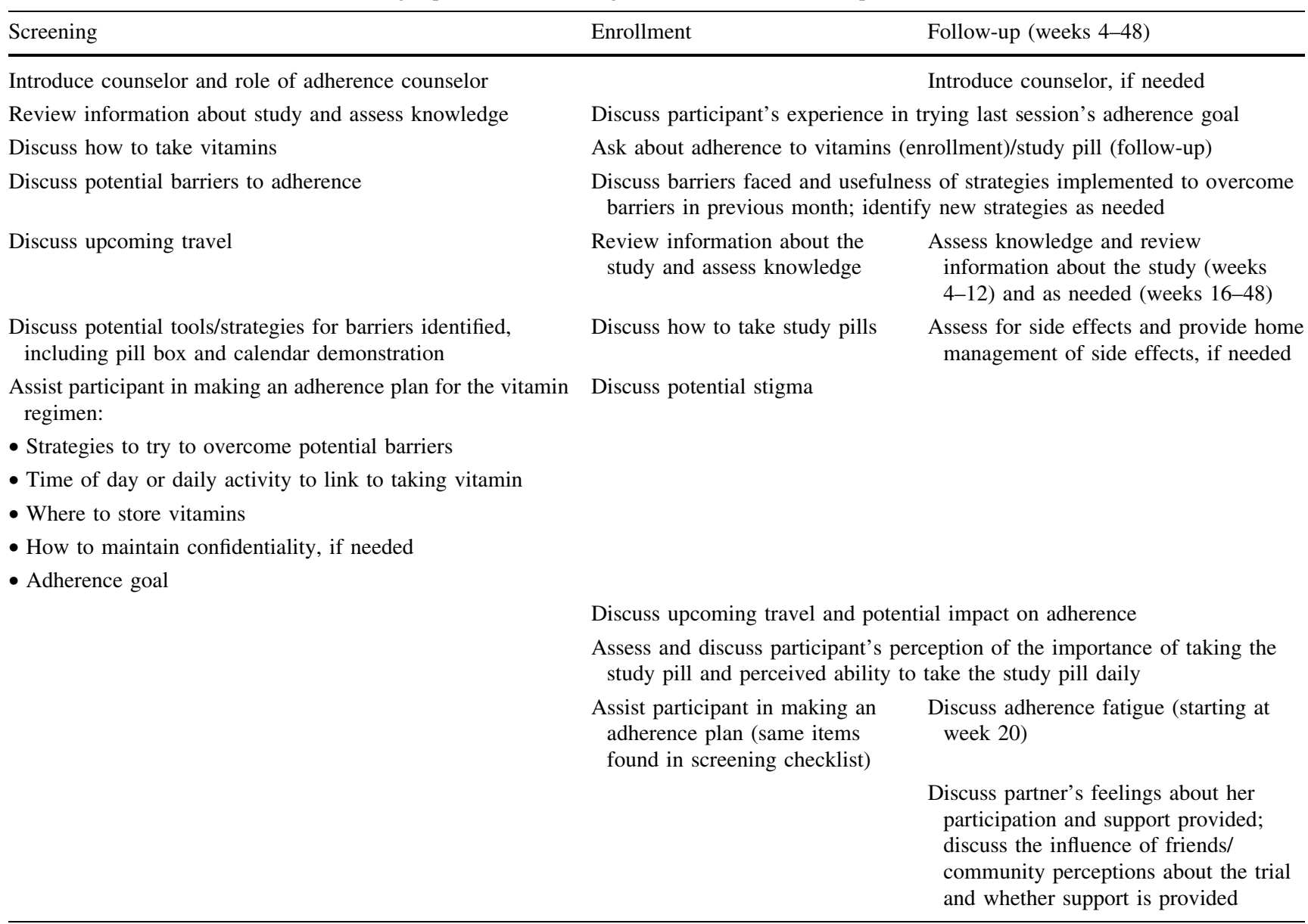

relationship dynamics, and motivation to join and remain in the trial.

\section{iPrEx RCT}

\section{Overview}

The iPrEx RCT investigated the safety and efficacy of daily oral TDF/FTC for HIV prevention among men who have sex with men (MSM) and transgendered women (TW) in Brazil, Ecuador, Peru, South Africa, Thailand, and the U.S. (see Table 1). Results from this phase III trial demonstrated an overall $44 \%$ reduction in HIV infection for TDF/FTC at intent to treat primary analysis [3], and $42 \%$ at extended follow-up, which covered an additional 6 months [31]. Among those who had drug levels detected, efficacy was above $90 \%$ [3, 32]. At the onset of the trial in July 2007, adherence to daily product use was well-recognized as a critical factor. Given that sites were highly diverse in location and culture, each site was asked to develop their own approach to adherence support, and site-level adherence via self-report was monitored monthly for feedback by the core research team. After 12-24 months from study start, site team members voiced concerns over potential 'pill-dumping' (where participants intentionally removed remaining pills prior to returning pill bottles to the study site in order to appear adherent), generally suggesting that product-use was not as high as self-report and productreturn-based adherence measures suggested.

An adherence working group (AWG) was formed to investigate the potential discrepancy between the measurement of study product use and its actual use, and to offer potential recommendations. Intervention mapping $[33,34]$ was used to gather and organize input from sites, participants, the literature, and experts. This mid-trial needs analysis suggested that sites were diverse in their approaches to supporting study product use, ranging from client-centered approaches to provision of informationonly and/or intensive messaging focusing on "perfect" rates of product use. Some sites also incorporated pill counts and results from interviewer-administered 
adherence measurements in their approach (where those identified as non-adherent had more intensive messaging and those with perfect adherence were praised and reinforced). Conversations about product-use stressed the need for taking the daily dose at a specific time, and prescribed strategies to achieve this regularity. Tools were also provided to promote daily product use, including pill cases, where available, and key fobs or dose-carrying tools. Adherence rates from product count and self-report were fed back to sites as a method of monitoring site performance. The AWG identified several areas for targeted improvement, and addressed these through the development of Next Step Counseling (NSC) to support product use and Neutral Assessment (NA) to assess self-reported product use via interviews [35].

\section{Adherence Support Approach: Next-Step Counseling/} Neutral Assessment

Drawing from results of the intervention mapping process [33], NSC and NA were developed by the AWG based on IMB models $[16,36]$ that were articulated to product use and to promoting accurate reporting of product non-use. NSC was advised by a number of intervention approaches, including risk-reduction, discussion-based approaches (e.g., the Options Project [18] and RESPECT [37]interventions), CAPRISA's ASP, MI [38], and clientcentered counseling. The steps and framing of NSC are presented in Fig. 2. The conversation is not scripted, neither is the content of a given conversation prescribed. The approach is designed with flexible content to be culturally adaptive and responsive to individual needs. The NSC approach recommends a general flow for product-use discussions and a climate to establish open conversation, while the specific wording and guidance that the counselors use are entirely individualized and make use of locally available resources. In developing NSC, the need for a counseling approach that could progress independent of reported non-adherence or difficulty with product use was prioritized, as cultural norms and the history of persuasion used to promote adherence at many sites made the accurate reporting of either unlikely. Additionally, NSC was assigned to one study team member, so that discussions of pill-use experiences were not diffused across multiple team members over the course of a single study visit. Thus, the NSC conversation focuses on participant experiences without outward attempts to extract problems to "fix." NA was adopted for the interviewer-administered adherence measurements where a non-NSC study team member conducted the interview. Any praise for reported high rates of product use or probing to resolve adherence "problems" were removed from the interviewer-administered adherence measurements.

\section{Monitoring and Evaluation}

The feasibility and acceptability of NSC and NA from the perspective of site implementers has been evaluated [35] and found to be largely positive. In practice, the NSC conversations appeared to be easily managed within the flow of study visits and were less intensive and shorter than the previous approach. Evaluation of the effectiveness of the NSC/NA approach is limited by its implementation late in the trial, after 34 months from initial launch of the study (see Table 1), with no control condition to adjust for the influence of elapsed time on study. Analyses of qualitative data exploring participant perceptions and acceptability of the NSC approach are ongoing. Moving forward, the iPrEx AWG has developed an integrated adherence counseling approach that jointly targets promoting PrEP use and other behavioral strategies to prevent HIV-infection. This approach, integrated Next Step Counseling (iNSC), is being used for the ongoing iPrEx open label extension (iPrEx OLE) study, where former iPrEX participants who have remained HIV-negative are provided the opportunity to receive PrEP, and HIV-positive participants are offered continued monitoring for 72 weeks.

\section{VOICE}

\section{Overview}

VOICE was a Phase IIb trial evaluating the safety and effectiveness of daily use of $1 \%$ tenofovir gel, oral tenofovir, and oral TDF/FTC for the prevention of HIV infection among sexually active, HIV-uninfected women in South Africa, Uganda, and Zimbabwe (see Table 1). The trial differs from CAPRISA 004 in that the prescribed dosing strategy for tenofovir gel was daily rather than coitally dependent. In September 2011, the oral tenofovir arm was stopped for futility [10]. This was followed by early stopping of the tenofovir gel arm in November 2011, when the Data Safety Monitoring Board reviewed data showing that the HIV incidence rates in the tenofovir gel and placebo gel groups were nearly identical (6.1 per 100 women-years in the placebo gel group and 6.0 per 100 women-years in the tenofovir gel group) [9]. The VOICE study is now completed, with the oral TDF/FTC and oral placebo arms ending on schedule in August 2012; full analyses of trial results are pending.

The adherence approach adopted at study start and implemented through the first 20 months of the trial was designed to be participant-centered and focused on 
Fig. 2 Steps and framing of NSC
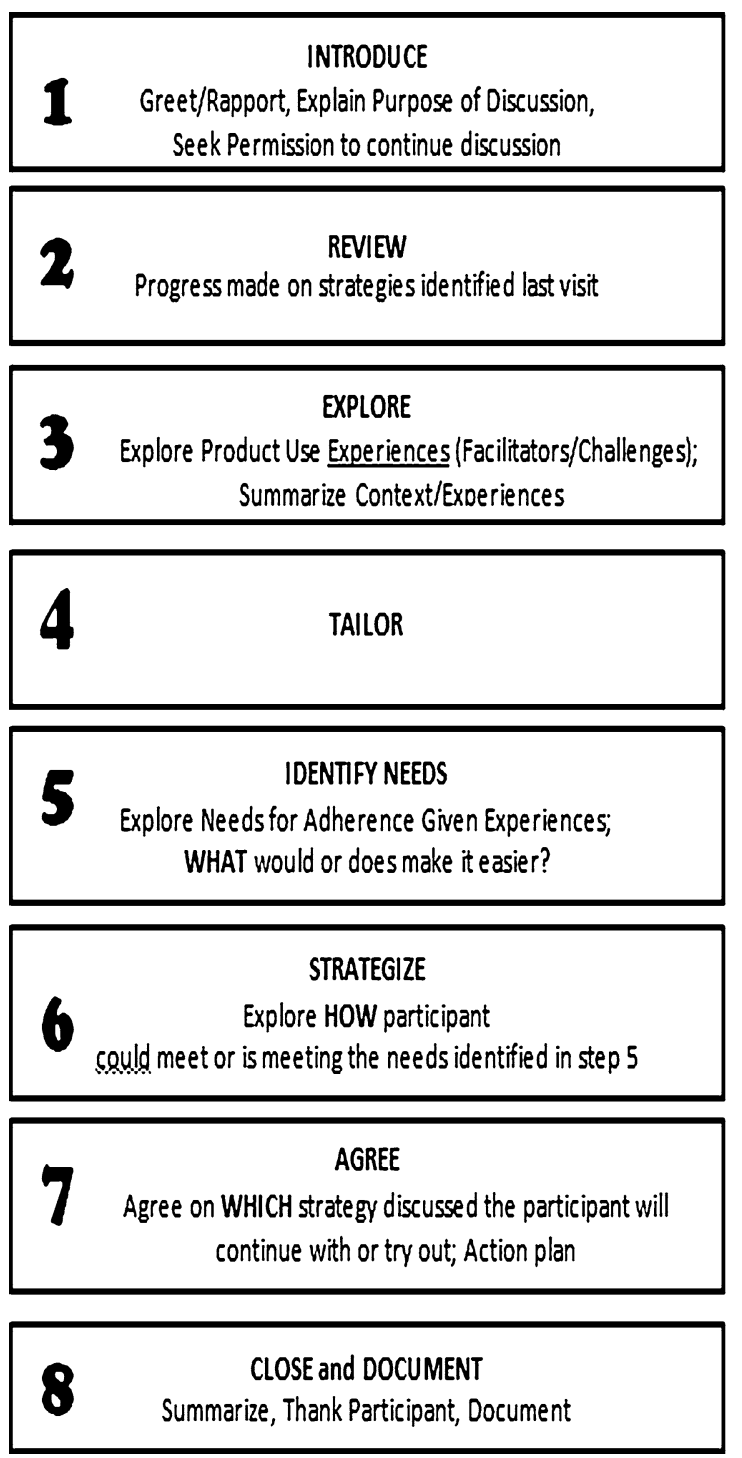

GOAL: Create a comfortable environment to talk about experiences with the product

CLIMATE: Supportive, nonjudgmental, neutral, reinforcing of open discussion/efforts, avoidance of "fixing," recognition of limited role, and emphasis on participant as a whole person.

\section{METHOD: Exploration of context} (experiences, thoughts, beliefs, feelings; information, motivation, skills) to identify needs and promote movement towards building a context that supports product use.

IMPLICITASSUMPTION:

Participants choose whether or not, or how much, to use the study product. We cannot make them use it, but can support open frank discussions about it. estimated levels of product adherence, based on both participant self-report (during the counseling session) and pharmacy accountability reports (provided to the counselor) of unused product returns. Counselors used scripts and worksheets to facilitate the counseling, which focused on overcoming barriers to perfect adherence. When product returns suggested perfect adherence, adherence counseling was brief and focused on congratulating the participant (positive reinforcement). High but not perfect adherence elicited a hybrid approach, where participants were congratulated and asked to discuss potential strategies to avoid missed doses in the future. Participants were provided with 10 key product-use messages at enrollment, and these were reviewed (as needed) at each visit. Key messages included guidance on what to do if you miss a dose, storage requirements, keeping product in one's possession, and two messages to take product daily.
While self-reported adherence appeared very high in VOICE based on initial reviews by the study team, concerns emerged in 2010, as the empirical evidence and reports from other trials increasingly suggested low actual adherence to daily biomedical prevention products. Feedback from VOICE site staff suggested that the estimated levels of adherence (based on unused product returns and self-report) may be inflated, and the current adherence support approach may not be offering sufficient opportunity for open conversations or discussions about actual product use. Based on feedback from site investigators and staff, and recommendations from outside experts, ${ }^{2}$ an

\footnotetext{
2 The MTN Behavioral Research Working Group (BRWG), sponsored by NIMH, convened an adherence meeting in September 2010 in Washington, DC with representatives from several key PrEP and microbicide trials, as well as adherence experts in the field.
} 
AWG was formed, which was tasked with revising and strengthening the adherence counseling approach in VOICE.

\section{Adherence Support Approach: VOICE Adherence} Strengthening Program (VASP)

Through an informal needs assessment and implementation of an intervention mapping process, the AWG adopted a revised strategy to support product use, entitled the VOICE Adherence Strengthening Program (VASP). The most critical changes were the elimination of counseling scripts targeting estimated adherence levels, and the elimination of product reconciliation between the pharmacy and selfreport during the counseling session. As such, VASP shifted the focus from adherence level to participant experience with product use. VASP was implemented midtrial (month 20 from study launch; see Table 1).

As can be seen from Fig. 3, VASP draws from NSC but uses some of the terminology from the original adherence support approach in the VOICE study to simplify its adoption by staff. Like the other trial adherence approaches reviewed here, the underlying model adopted to identify potential core determinants of product use, as well as comfort around discussing use, was based on the IMB model [16, 36], MI [38], and client-centered counseling. Additionally, working with women in many communities with diverse needs and experiences heightened the importance of a brief, flexible, and non-prescriptive intervention. VASP focuses on women's experiences and needs, in order to integrate product use into daily life through brief monthly conversations with a VOICE adherence counselor. Like NSC, it focuses on adherence-related needs (instead of barriers) and is relevant to all participants, regardless of their level of product use. VASP promotes a neutral atmosphere designed to facilitate comfort in speaking openly about experiences with product use and non-use.

\section{Monitoring and evaluation}

Process monitoring of VASP implementation included monthly or bi-monthly calls facilitated by FHI360 and behavioral scientists involved in the trial and development of VASP where one of two "mentors" (designated nurse counselors or counselors from each site) joined the call to share concerns or observations from their site. Three waves of anonymous web-based surveys with counseling staff (pre-training, 6-month, and end of trial), as well as IDIs with a subset of counselors at selected sites, were conducted. Interviews with a subset of exited VOICE participants are also planned. Drug level analyses of stored biological specimens will be conducted to characterize adherence among study participants assigned to the active arms in VOICE. When available, drug exposure data may allow a more formal estimation of actual adherence during the pre- and post-VASP portions of the trial. Unfortunately, the early discontinuation of two of the study products due to futility shortly after VASP implementation will limit the ability to fully assess the potential effect that VASP may have had on participant behavior.

\section{Summary and Recommendations}

Across the approaches reviewed in the four oral and microbicide gel PrEP trials, the critical role of adherence was recognized from study onset, with promotion and monitoring strategies in place from the start. However, few trials have fully appreciated the more nuanced impact of social, individual, and clinic-related factors that would ultimately influence product-use and the accurate reporting of it. Rather, in many cases trials started with a strong emphasis on education, information, and use of persuasion (praise, reinforcement, accountability) to promote "perfect" adherence. Modifications to varying degrees were made in several of the trials to move beyond information-provision and optimize adherence support using well-established health behavior models and intervention strategies. All four approaches converged in regard to underlying health behavior theory, trainings deployed on approach, implementation at study visits, use of motivation-enhancement strategies, and brevity of adherence-support conversations.

Despite considerable consistency in the approaches used in these four different trials, estimated product use (where available) varied considerably and was generally lower than desired. Without an experimental design, it is not possible to evaluate if the enhanced adherence support approaches implemented had any effect on actual product adherence. Because several trials changed approaches to supporting and/or monitoring adherence mid-trial or later, evaluation of the effect of these changes is particularly complicated. Any attempt to evaluate the impact of the adherence approach or subsequent changes would have to account for multiple sources of bias (e.g., dropping of specific arms in a study, release of CAPRISA 004 results for participants in other studies, increasing length of time on study, etc.) and this cannot be done without a nonadherence intervention comparison group. As such, the added benefit of any given approach will be difficult to conclusively evaluate. Qualitative data collected in the context of these trials are under evaluation, which will provide much needed guidance on the role of socio-cultural and community based factors as contributors to (non)adherence. Diversity in participant populations, risk-perception, culture, community, structures promoting and inhibiting product use, and the culture of sites themselves 
Fig. 3 Steps and framing of VASP

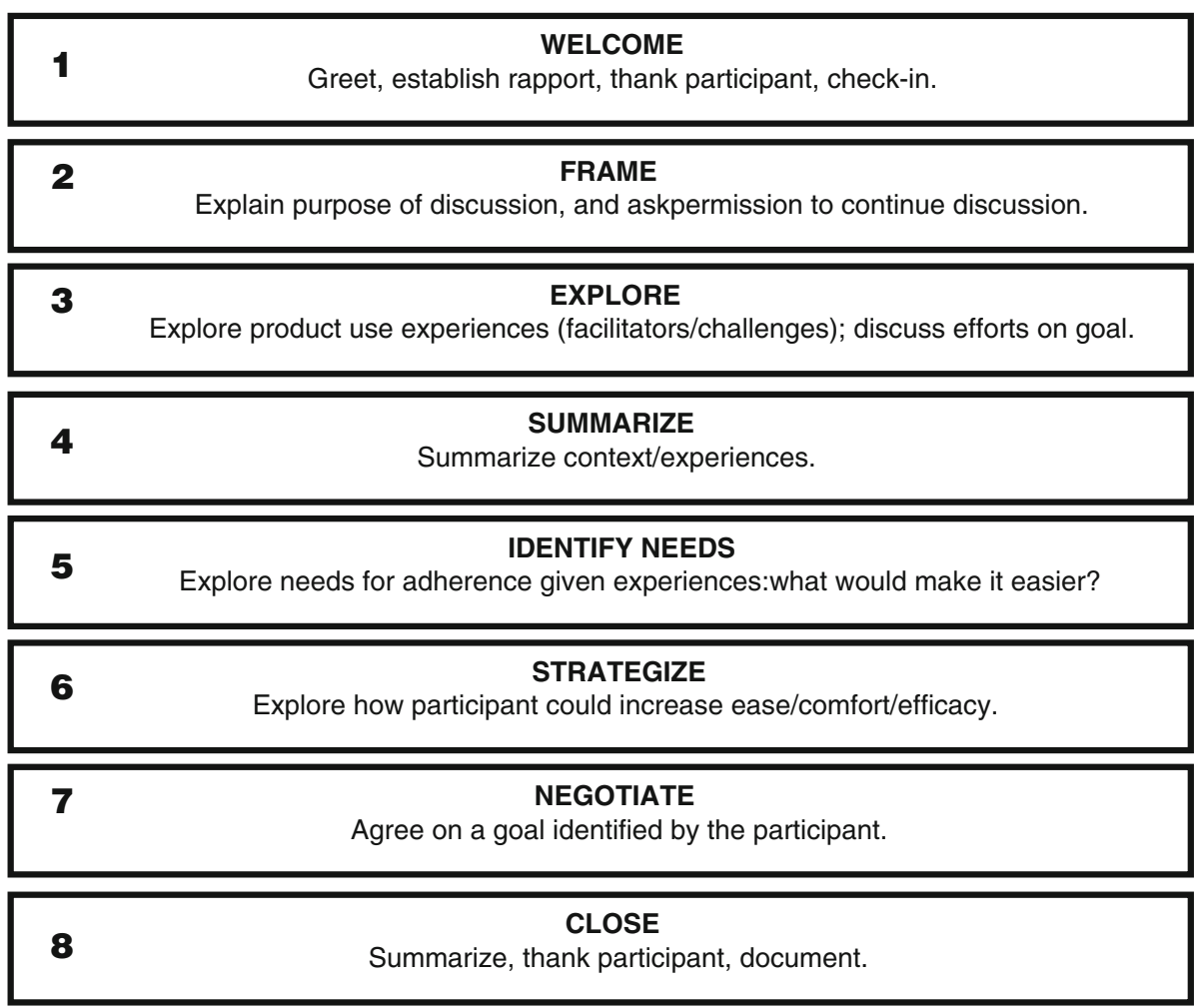

may likely explain a large part of the variability reported in product use across trials, sites, and participants. These social-cultural dynamics need targeted exploration and research to fully appreciate the context in which regimens are presented, "prescribed," executed, and maintained. A more thorough exploration of these dynamics can take place through implementation of high quality behavioral research in the context of PrEP demonstration projects, including examination of the models developed to guide the intervention approaches (e.g., what are the main drivers of adherence to PrEP or to product use, how do they interrelate, and what proportion of variability in adherence do they reliably explain), as well as through randomized controlled trials of specific behavioral intervention strategies to promote PrEP or product use.

While most trials reviewed here documented to some extent the implementation of adherence support approaches and exploration of general acceptability and feasibility of the approaches, none used fidelity and implementation monitoring strategies commonly adopted in trials of behavioral interventions (e.g., video or audio review or evaluation of counseling sessions). Fidelity of implementation was often captured only at the most basic level, through collection of checklists or worksheets of steps implemented completed by counselors, leaving many questions concerning actual implementation unanswered. Even if adherence support approaches are well-matched to the participants and their socio-cultural surroundings, intervention drift, adaptation and modification will likely occur to varying degrees (cf., [39]). Indeed, allocation of resources and competing trial priorities limited the feasibility of intensive monitoring of implementation in any of the trials discussed here. While each trial can speak to what was recommended and how adherence support staff were trained, the fidelity of what was actually implemented and the extent to which it modeled participant-centered approaches versus instruction and persuasion is not clear. Research employing methods to fully characterize implementation and fidelity of adherence support approaches (e.g., use of systematic evaluation of implementation through observation, audio or visual recording, and content, process, or discourse analyses) is needed.

With these limitations in mind, it is critical to add rigorous behavioral research to the agenda for PrEP demonstration and on-going efficacy trials. Partners PrEP [2], a phase III double-blind, placebo controlled, clinical trial of safety and efficacy of oral PrEP (TDF alone or in combination with FTC) among serodiscordant couples in Kenya and Uganda, is one of the only studies completed to date that specifically included a structured adherence intervention as a sub-study. The sub-study involved over a thousand sero-discordant couples at three of the nine parent study sites and used objective measures of product-use to both trigger and evaluate intervention [40]. Results from this sub-study in terms of effects on product use are expected in 2013 and will provide guidance on the use of 
targeting adherence support (e.g., providing support when product-use is identified as sub-optimal) and the adaption of a demonstrated treatment intervention to support PrEP adherence (LifeSteps, described in [41]). Results supporting near-perfect adherence among Partners PrEP participants have already been reported [2, 40], as have the potentially unique motivations for product use within serodiscordant partnerships [42]. Of particular importance, Partners PrEP demonstrates the feasibility of clinical trial designs that include comprehensive monitoring of product use and implementation of rigorous behavioral interventions. The Partners PrEP example shows that these designs are not only feasible, but also generate interpretable results and provide a better understanding of the relative contribution of the behavioral and biomedical components of PrEP trials to the overall study findings.

Shifting from a biomedical to a bio-behavioral, or rather a bio-psycho-social, framework will help build the evidence base for effective PrEP adherence interventions. As we enter a new era with oral PrEP now available in some locations [43], shifting attention to support open label PrEP use among those who seek out and adopt this prevention strategy will offer new insights and opportunities to carefully consider the role of the individual, partners, community, care providers, health systems, and the dosing regimen in the ultimate success of PrEP.

Acknowledgments This manuscript greatly benefited from the editorial and content contributions from Quarraisha Abdool Karim. We also wish to acknowledge the valuable reviews from Vanessa McMahan, iPrEx RCT, and reviewers from MTN. All four study teams would like first and foremost to pay tribute to the women and men who participated in these trials, their dedication and commitment made these studies possible. CAPRISA 004: The CAPRISA 004 tenofovir gel trial was supported by the Centre for the AIDS Program of Research in South Africa (CAPRISA), the United States Agency for International Development (USAID), FHI (co operative agreement \# GPO-A-00-05-00022-00, contract \# 132119), and LIFElab, a biotechnology centre of the South African Department of Science and Technology. Support from CONRAD for the product manufacturing and packaging as well as support from Gilead Sciences for the tenofovir used in the production of gel is gratefully acknowledged. We thank the US National Institutes for Health's Comprehensive International Program of Research on AIDS (CIPRA grant \# AI51794) and the Columbia University-Southern African Fogarty AIDS International Training and Research Programme (AITRP grant \# D43TW00231) for the research infrastructure and training that made this trial possible. FEM-PrEP: The FEM-PrEP team would like to acknowledge and thank Judith Harkins for developing the adherence counseling session program and materials. FEM-PrEP was a partnership between FHI 360 in the U.S. and local scientists and project staff at the Impact Research and Development Organization (Kenya), Setshaba Research Centre (Pretoria, South Africa), Josha Research (Bloemfontein, South Africa), and Kilimanjaro Christian Medical Center (Tanzania). FEM-PrEP Investigators included Drs. Lut Van Damme (Clinical PI), Amy Corneli (Behavioral PI), Kawango Agot (Bondo Site Investigator), Khatija Ahmed (Pretoria Site Investigator), Johan Lombaard (Bloemfontein Site Investigator), and Rachel Manongi (Arusha Site Investigator). The study was funded by the U.S.
Agency for International Development (USAID), and it also received early support from the Bill \& Melinda Gates Foundation. iPrEx: The contributions of the Adherence Working Group, Ed Wolf, and the study site team members are acknowledged as critical factors in the development and implementation of NSC and NA. The full iPrEx study team can be viewed at http://iprexole.com/. The iPrEx RCT was funded by the National Institutes of Health and the Bill and Melinda Gates Foundation; ClinicalTrials.gov number, NCT00458393. VOICE: The contributions of the MTN Behavioral Research Working Group, the VOICE leadership, the VOICE Adherence Working Group, Ashley Mayo of FHI360, Ed Wolf, and the study site investigators and team members are acknowledged as critical factors in the development and implementation of VASP. MTN is funded by NIAID (5UM1AI068633), NICHD and NIMH, all of the U.S. National Institutes of Health. The full VOICE study team can be viewed at http://www.mtnstopshiv.org/news/studies/mtn003.

\section{References}

1. Abdool Karim Q, Abdool Karim SS, Frohlich JA, Grobler AC, Baxter C, Mansoor LE, et al. Effectiveness and safety of tenofovir gel, an antiretroviral microbicide, for the prevention of HIV infection in women. Science. 2010;329(5996):1168-74.

2. Baeten JM, Donnell D, Ndase P, Mugo NR, Campbell JD, Wangisi J, Tappero JW, Bukusi EA, Cohen CR, Katabira E, Ronald A, Tumwesigye E, Were E, Fife KH, Kiarie J, Farquhar C, JohnStewart G, Kakia A, Odoyo J, Mucunguzi A, Nakku-Joloba E, Twesigye R, Ngure K, Apaka C, Tamooh H, Gabona F, Mujugira A, Panteleeff D, Thomas KK, Kidoguchi L, Krows M, Revall J, Morrison S, Haugen H, Emmanuel-Ogier M, Ondrejcek L, Coombs RW, Frenkel L, Hendrix C, Bumpus NN, Bangsberg D, Haberer JE, Stevens WS, Lingappa JR, Celum C, the Partners PrEP Study Team. Antiretroviral prophylaxis for HIV prevention in heterosexual men and women. N Engl J Med. 2012;367(5):399-410.

3. Grant RM, Lama JR, Anderson PL, McMahan V, Liu AY, Vargas $\mathrm{L}$, et al. Preexposure chemoprophylaxis for HIV prevention in men who have sex with men. N Engl J Med. 2010;363(27):2587-99.

4. Thigpen MC, Kebaabetswa PM, Paxton L, Smith DK, Rose CE, Segolodi TM, Henderson FL, Pathak SR, Soud FA, Chillag K, Mutanhaurwa R, Chirwa LI, Kasonde M, Abebe D, Buliva E, Gvetadze R, Johnson S, Sukalac T, Thomas VT, Hart C, Johnson JA, Malotte CK, Hendrix CW, Brooks JT, for the TDF2 Study Group. Antiretroviral preexposure prophylaxis for heterosexual HIV transmission in Botswana. N Engl J Med. 2012. doi:10.1056/ NEJMoa1110711.

5. Van Damme L, Corneli A, Ahmed K, et al. Preexposure prophylaxis for HIV infection among African women. N Engl J Med. 2012;367(5):411-22.

6. Microbicide Trials Network (MTN). MTN statement on decision to discontinue use of Tenofovir gel in VOICE, a major HIV prevention study in women. 2011. Available at: http://www. mtnstopshiv.org/node/3909. Accessed Sept 252011.

7. Microbicide Trials Network. MTN statement on decision to discontinue use of oral Tenofovir tablets in VOICE, a major HIV prevention study in women. 2011. Available at: http://www. mtnstopshiv.org/node/3619. Accessed Sept 252011.

8. Kashuba AD, Patterson KB, Dumond JB, Cohen MS. Pre-exposure prophylaxis for HIV prevention: how to predict success. Lancet. 2012;379(9835):2409-11.

9. Stirratt MJ, Gordon CM. Adherence to biomedical HIV prevention methods: considerations drawn from HIV treatment adherence research. Curr HIV/AIDS Rep. 2008;5(4):186-92.

10. Karim S, Karim QA. Antiretroviral prophylaxis: a defining moment in HIV control. Lancet. 2011;378(9809):e23-5. 
11. Amico KR. Adherence to preexposure chemoprophylaxis: the behavioral bridge from efficacy to effectiveness. Curr Opin HIV AIDS. 2012;7(6):542-8.

12. Muchomba FM, Gearing RE, Simoni JM, El-Bassel N. State of the science of adherence to pre-exposure prophylaxis and microbicide trials. J Acquir Immune Defic Syndr. 2012;61(4):490-8.

13. Centers for Disease Control and Prevention. Morbidity and mortality weekly report (MMWR), Interim guidance: Preexposure prophylaxis for the prevention of HIV infection in men who have sex with men. 2011;60(03):65-68. Available at: http://www. cdc.gov/mmwr/preview $/ \mathrm{mmwrhtml} / \mathrm{mm} 6003 \mathrm{a} 1 . \mathrm{htm}$ ?s_cid=mm 6003a1_w. Accessed Sept 252011.

14. Centers for Disease Control and Prevention. Morbidity and mortality weekly report (MMWR), Interim guidance for clinicians considering the use of preexposure prophylaxis for the prevention of HIV infection in heterosexually active adults. 2012. Available at: http://www.cdc.gov/Mmwr/preview/mmwrhtml/ mm6131a2.htm?s_cid=mm6131a2_w. 9 Aug 2012.

15. World Health Organization. Guidance on oral pre-exposure prophylaxis (prep) for serodiscordant couples, men and transgender women who have sex with men at high risk of HIV: recommendations for use in the context of demonstration projects. 2012. Available at: http://www.who.int/hiv/pub/guidance_prep/ en/index.html. Accessed 9 Sept 2012.

16. Fisher JD, Fisher WA. Changing AIDS-risk behavior. Psychol Bull. 1992;111(3):455.

17. Karim QA, Kharsany AB, Naidoo K, Yende N, Gengiah T, Omar Z, Arulappan N, Mlisana KP, Luthuli LR, Karim SS. Coenrollment in multiple HIV prevention trials - experiences from the CAPRISA 004 tenofovir gel trial. Contemp Clin Trials. 2011;32(3):333-8

18. Fisher JD, Cornman DH, Osborn CY, Amico KR, Fisher WA, Friedland GA. Clinician-initiated HIV risk reduction intervention for HIV-positive persons: formative research, acceptability, and fidelity of the Options Project. J Acquir Immune Defic Syndr. 2004;37(Suppl 2):S78-87.

19. Rollnick S, Miller WR. What is motivational interviewing? Behav Cogn Psychother. 1995;23(4):325-34.

20. Emmons KM, Rollnick S. Motivational interviewing in health care settings: opportunities and limitations. Am J Prev Med. 2001;20(1):68-74.

21. Mansoor LE. Enhancing adherence to study product through motivational interviewing: Experiences from CAPRISA 004. Miami: Presented at the 6th International Conference on Treatment and Prevention Adherence; 2011.

22. Corneli AL, Mack N, Kirkendale S, editors. Socio-behavioral and community activities for an oral HIV prevention prep clinical trial: a multi-disciplinary approach. New Delhi: International Microbicides Conference; 2008.

23. FHI 360. Joint statement on savvy phase 3 trial in Ghana to test the effectiveness of savvy gel in preventing HIV. 2005. Available at http://www.fhi360.org/en/AboutFHI/Media/Releases/pr2005/ Nov082005GhanaSavvyTrial.htm. Accessed 9 Sept 2012.

24. FHI. VCT Toolkit: HIV voluntary counseling and testing: a reference guide for counselors and trainers. Arlington: Family Health International; 2004.

25. FHI. Adherence support worker training facilitator's guide. Arlington: Family Health International; 2007.

26. FHI. Nursing care of patients with HIV/AIDS: facilitator's guide. Arlington: Family Health International; 2008.

27. Halpern V, Lopez LM, Grimes DA, Gallo MF. Strategies to improve adherence and acceptability of hormonal methods of con-traception. status and date. Cochrane Database Syst Rev. 2011;. doi:10.1002/14651858.CD004317.

28. Blouse A, WAgner E. Counseling and testing for HIV: reference manual. Baltimore: JHPEIGO; 2004.
29. Munro S, Lewin S, Swart T, Volmink J. A review of health behaviour theories: how useful are these for developing interventions to promote long-term medication adherence for TB and HIV/AIDS? BMC Public Health. 2007;7(1):104.

30. Kidd R, Clay S. Understanding and challenging HIV stigma: toolkit for action. New York: CHANGE Project (AED) International Center for Research on Women. 2003. Available at http:// www.icrw.org/publications/understanding-and-challenging-hivstigma-toolkit-action. Accessed 17 Sept 2004.

31. Grant R, McMahan A, Liu J, et al. Completed observation of the randomized placebo-controlled phase of iPrEx: daily oral FTC/ TDF pre-exposure HIV prophylaxis among men and trans women who have sex with men. Sixth IAS Conference on HIV Pathogenesis, Tratement and PRevention, 2011 [abstract WELBC04]. Available at http://pag.ias2011.org/PAGMaterial/IAS2011/PPT/ 598_642/grant\%20et\%20al\%20completed\%20observation\%20 final\%204.pptx. Accessed on 25 Sept 2012.

32. Grant R, Lama J, Glidden D, iPrEx Study Team. Pre-exposure chemprophylaxis for prevention of HIV among trans-women and MSM: iPREx study. Boston: 18th Conference on Retroviruses and Opportunistic Infections (CROI); 2011.

33. Bartholomew LK, Parcel GS, Kok G, Gottlieb NH. Planning health promotion programs: an intervention mapping approach. 2nd ed. San Francisco: Jossey-Bass; 2006.

34. Kok G, Schaalma H, Ruiter RA, Van Empelen P, Brug J. Intervention mapping: protocol for applying health psychology theory to prevention programmes. J health Psychol. 2004;9(1):85-98.

35. Amico KR, McMahan V, Goicochea P, Vargas L, Marcus JL, Grant RM, et al. Supporting study product use and accuracy in self-report in the iPrEx study: next step counseling and neutral assessment. AIDS Behav. 2012;16(5):1243-59.

36. Fisher JD, Amico KR, Fisher WA, Harman JJ. The InformationMotivation-Behavioral Skills model of antiretroviral adherence and its applications. Curr HIV/AIDS Rep. 2008;5(4):193-203.

37. Kamb ML, Fishbein M, Douglas JM Jr, Rhodes F, Rogers J, Bolan G, et al. Efficacy of risk-reducing counseling to prevent human immunodefiency virus and sexually transmitted diseases. JAMA. 1998;280(13):1161-7.

38. Rollnick S, Miller WR, Butler CC. Motivational interviewing in health care: helping patients change behavior. New York: Guilford Press; 2008.

39. Norton WE, Amico KR, Cornman DH, Fisher WA, Fisher JD. An agenda for advancing the science of implementation of evidencebased HIV prevention interventions. AIDS Behav. 2009;13(3):424-9.

40. Harberer J, Baeten J, Celum C, Katibira E, Ronald, et al. High adherence among HIV-1 serodiscordant couples in the Partners PrEP ancillary adherence study. Miami: International Association for Physicians in AIDS Care. 2012. Available at: http://www. iapac.org/AdherenceConference/presentations/ADH7_80026.pdf. Accessed on 9 Sept 2012.

41. Psaros C. evaluation and process outcomes from an adherence intervention to support HIV pre-exposure prophylaxis (PrEP) adherence in HIV-serodiscordant couples in Uganda. Miami: Oral presentation at the 7th Annual HIV Treatment and Prevention Adherence Conference of the International Association of Providers in AIDS Care. 2012. Available at http://www.iapac.org/AdherenceConference/ presentations/ADH7_80031.pdf. Accessed on 20 Sept 2012.

42. Ware NC, Wyatt MA, Haberer JE, Baeten JM, Kintu A, Psaros C, Safren S, Tumwesigye E, Celum CL, Bangsberg DR. What's love got to do with it? Explaining adherence to oral antiretroviral preexposure prophylaxis for HIV-serodiscordant couples. J Acquir Immune Defic Syndr. 2012;59(5):463-8.

43. US Food and Drug Administration. FDA approves first drug for reducing the risk of sexually acquired HIV infection. 2012. Available at: http://www.fda.gov/NewsEvents/Newsroom/Press Announcements/ucm312210.htm. Accessed on 9 September 2012. 